\title{
Análisis de las estrategias para la prevención de la deserción y retención de la población estudiantil que lleva a cabo el personal docente y administrativo del Liceo de Miramar, de la Dirección Regional de Educación de Puntarenas, Costa Rica
}

\author{
Analysis of strategies executed by Miramar's High School Teachers and Staff, from the Regional Board of \\ Education of Puntarenas, Costa Rica, to Prevent Desertion and Students Population Retention
}

Rodolfo Ovares Elizondo'

Ministerio de Educación Pública

rodolfoovares@gmail.com

Recibido 10 junio 2012 • Aceptado 25 noviembre 2012 • Corregido 29 noviembre 2012

\begin{abstract}
Resumen. En este artículo se analiza la deserción escolar como el flagelo que afecta a la sociedad actual, tanto que provoca pérdidas cuantiosas y, con el paso del tiempo, amplía las brechas sociales como consecuencia del abandono de la educación por parte de la población estudiantil. En este sentido se desarrolló una investigación tipo descriptivo apoyado con técnicas cuantitativas y cualitativas en el Liceo de Miramar de Puntarenas, para el análisis de las estrategias de prevención de la deserción escolar y retención estudiantil, desde la perspectiva del personal docente y administrativo.

El estudio pretende determinar los factores que provocan la deserción estudiantil, las estrategias que aplica el personal docente y administrativo desde la gestión de la educación y la percepción de la eficacia y eficiencia de esas acciones para la prevención de la deserción y la retención estudiantil en el centro educativo. Asimismo, generar un compromiso por parte de la comunidad educativa para el trabajo conjunto en torno a este fenómeno presente en la educación costarricense.
\end{abstract}

Palabras Claves. Administración de la educación, deserción estudiantil, retención escolar, estrategias para la prevención de la deserción estudiantil.

Abstract. This article analyzes the dropout as the scourge affecting today's society, while causing significant losses and, over time, widens social gaps as a result of dropping out of school by the student population. Thus we developed a descriptive research supported with quantitative and qualitative techniques in the Liceo de Miramar de Puntarenas, to analyze strategies dropout prevention and student retention, from the perspective of faculty and staff.

The study aims to determine the factors causing dropout, the strategies employed by faculty and staff from the management of education and perception of the efficiency and effectiveness of those actions for dropout prevention and student retention in school. Also generate a commitment from the education community to work together on this phenomenon in Costa Rican education.

Keywords. Management education, dropout, retention, strategies for dropout prevention.

\footnotetext{
Máster en Ciencias de la Educación con énfasis en Administración Educativa de la Universidad de Costa Rica. Bachiller y licenciado en la
} Enseñanza de la Matemática de la Universidad Nacional .Se desempeña como docente en el Liceo de Miramar, Puntarenas, Costa Rica. 


\section{Introducción}

En la constante búsqueda de la calidad educativa en la sociedad actual, existe la necesidad de enfrentar diversos factores que se relacionan con la deserción estudiantil. Algunos de ellos enfatizan aspectos socioeconómicos, contextuales y los vinculados al currículo (Ruiz, 2006); otros por su parte, indican el bajo rendimiento, la repetición y los problemas de conducta (Espíndola y León, 2002). Un común en este sentido, es el abandono estudiantil que adquiere mayores dimensiones, debido al hecho de que los sistemas educativos latinoamericanos poseen escasa capacidad de retención de la niñez y particularmente, de jóvenes, según la Organización de Estados Americanos (OEA, 2003).

Por mandato de la sociedad, la administración de la educación está en la obligación de diseñar y ejecutar procesos teóricos y prácticos para el mejoramiento del sistema educativo (Pozner, 2000), lo que incide en la formación integral del ser humano mediante la administración del currículo en un tiempo y espacio requerido, a partir del manejo cognitivo, actitudes y valores que deben ser planificados, organizados, dirigidos y evaluados, con participación de todos los sujetos e instancias involucrados en el proceso (Venegas, 2001).

Así, la administración de la educación como proceso orienta el quehacer educativo en el desarrollo de un país, por lo cual ha de considerar, entre las diversas problemáticas que se presentan en la sociedad actual, el fenómeno de la deserción, con el firme propósito de alcanzar las metas y objetivos organizacionales. Al respecto, Vargas (2008) expresa que los profesionales en la administración de la educación suponen una deliberación y toma de decisiones responsable, para facilitar la construcción de una comunidad educada y capaz de establecer un orden dinámico y democrático.

La deserción escolar interviene en la construcción de esta comunidad, ya que afecta el sistema educativo y con ello, a toda una nación. La ausencia de la población estudiantil en las aulas aumenta la amenaza de un porvenir académico truncado y a medias, por parte de la juventud, acarreando consigo consecuencias en sus vidas y en la vida social del país en que viven, mismo que ha sido forjado bajo el fundamento de la educación (Instituto de Estudios Sociales en Población, 2006), lo que ha permitido una ventaja sobre muchos países de Latinoamérica.

En el caso de Costa Rica, la situación no es diferente. Por el contrario, la idiosincrasia del ser costarricense, determina la educación como un derecho y una cuestión de equidad que perjudica, en mayor medida, al sexo femenino más que al masculino, a la zona rural más que a la urbana, a los pobres más que a los ricos y a las minorías étnicas más que a las mayorías (Programa Estado de la Nación en Desarrollo Humano Sostenible, 2008).

Asimismo, en el Informe Estado de la Nación (Programa Estado de la Nación en Desarrollo Humano Sostenible, 2008) se manifiesta que, en materia presupuestaria, la deserción y la 
reprobación estudiantil le cuestan al Estado cerca de 51.000 millones de colones por año, lo que representa aproximadamente un $0,5 \%$ del PIB; además, la inversión general en educación, representa el $37,5 \%$ de la estimación de la distribución del gasto del Gobierno.

En este sentido, el Ministerio de Educación Pública (MEP) realiza esfuerzos para reducir la deserción que se presenta en todos los niveles escolares, a partir de la importancia que tiene la educación para el Estado, reforzando las ayudas económicas de los escolares por medio de programas educativos como "Avancemos", e implementando medidas como el adelantamiento de materias a nivel de secundaria, en busca de minimizar los efectos que, a largo plazo, tendrán repercusiones en los ámbitos económico, social y cultural del país.

En el nivel micro del sistema educativo, resulta importante que la administración de la educación establezca prácticas para el control de la deserción en los centros educativos, lo cual exige herramientas que incidan, directamente, en la reducción de los índices de deserción, en beneficio de la población estudiantil y por ende, de sus familias, sobre todo de aquellas que están en riesgo de sufrir la deserción educativa.

La deserción escolar es el flagelo que afecta a la sociedad, tanto que provoca pérdidas cuantiosas y, con el paso del tiempo, ensancha las brechas sociales como consecuencia del abandono de la educación por parte de la población estudiantil.

Al respecto, Cardona (2002), citado por Castro y Rivas (2006), le da una gran magnitud a la deserción al expresar que mucha de la juventud deserta antes de irse físicamente del centro educativo, puesto que han desertado del conocimiento; a su vez, muchos de los que se quedan en la institución, lo hacen por ser el lugar principal de encuentro con sus pares.

Si bien es cierto, la deserción es la problemática de fondo, también cabe determinar el papel de la administración de la educación en ese proceso en los centros educativos, principalmente, por ser esta la responsable de proporcionar las herramientas para el buen desarrollo del currículo educativo en una institución determinada.

Toda acción diseñada para disminuir el índice de deserción es necesario que asegure el desarrollo y el compromiso humano dentro de una adecuada estructura organizacional (Rojas, 2000). Generar compromisos de los miembros de la comunidad educativa, trabajo conjunto donde estén claras las responsabilidades de cada uno de ellos, para ello se debe conocer el fenómeno, características, consecuencias e informar a los miembros dichos hallazgos.

A partir del problema descrito, la investigación responde a los siguientes objetivos, los cuales orientan el estudio que se realiza en relación con las estrategias para la prevención de la deserción escolar y retención de la población estudiantil en el Liceo de Miramar, Puntarenas. 


\section{Objetivos generales}

1. Analizar las estrategias para la prevención de la deserción y retención de la población estudiantil que lleva a cabo el personal docente y administrativo del Liceo de Miramar, de la Dirección Regional de Educación de Puntarenas.

2. Diseñar una propuesta de gestión para el fortalecimiento de las estrategias para la prevención de la deserción y retención de la población estudiantil en el Liceo de Miramar a partir de la acción del personal docente y administrativo.

\section{Objetivos específicos}

1.1 Identificar los factores que propician la deserción escolar en el Liceo de Miramar desde el punto de vista del personal docente y administrativo.

1.2 Establecer las estrategias para la prevención de la deserción y retención de la población estudiantil, que llevan a cabo el personal docente y administrativo del Liceo de Miramar.

1.3 Identificar la eficacia de las estrategias para la prevención de la deserción y retención de la población estudiantil que el centro educativo aplica.

2.1. Elaborar estrategias de gestión para el control de la deserción escolar en el Liceo de Miramar

\section{Marco de referencia}

Hoy día, los centros educativos ofrecen a la sociedad una serie de servicios que son trascendentales para la formación del ser humano. Por esa razón, se hace necesario que las organizaciones sean eficaces y ágiles en el cumplimiento de sus funciones.

La funcionalidad de toda organización se encuentra en la dirección, particularmente, en las decisiones o acciones que se ejecutan a través de criterios científicos. De ahí, que en este estudio se entienda la administración como el "proceso de alcanzar las metas de la organización, trabajando con y por medio de la gente y de otros recursos organizacionales" (Certo, 2001, p. 6), lo que implica necesariamente las interacciones con personas y el buen manejo de los recursos.

Visto como parte de ese funcionamiento organizacional, se requiere contar, además, con un diseño que colabore en el mantenimiento de un ambiente donde individuos, que trabajan juntos en grupos, cumplan metas específicas de manera eficiente (Kootz, Weihreich y Cannice, 
2008). Es indudable, por tanto, que las organizaciones actuales potencien el trabajo grupal y los recursos que pueden generar, por medio de herramientas que faciliten el alcance de esos proyectos, de manera que los esfuerzos y recursos se utilicen de la mejor manera.

Por su parte Venegas (2001) define la administración de la educación como la "ciencia y arte que busca el logro de políticas, objetivos y propósitos educativos mediante el desarrollo de instituciones y programas que fomentan la adquisición de conocimientos, actitudes y valores humanos, sustentados en el currículo institucional, comunitario o regional, nacional y universal" (p. 4). En esta dirección, se le asigna la responsabilidad en el diseño de acciones que otorguen agilidad, que permitan controlar y responder a los problemas que se presentan a diario en el aula y fuera de ella, con la participación de los diferentes actores involucrados en el proceso educativo (Duchi y Andrade, 2001).

La búsqueda de los objetivos propuestos como deben ser el bienestar y formación del estudiante implica una constante valoración y modificación de acciones diseñadas para tal fin, por lo cual es necesario que la administración de la educación plantee verdaderos procesos donde se formule, implemente y evalúen las decisiones interfuncionales que tienen su origen entre lo estratégico y operativo (Arroyo, 2007).

\section{Papel del profesional en administración de la educación.}

La dirección de cualquier institución implica la participación de alguien como responsable de las decisiones que se toman o se dejan de tomar en la organización. No obstante a su autoridad la conducción escolar se presenta como algo difícil y comprometido, lo cual requiere de la profesionalización de la persona que ejerce la dirección con respecto a habilidades y conocimiento referidos a las dimensiones pedagógicas, en la organización de sus funciones y en las acciones económica-administrativa del proyecto institucional (García, Rojas y Campos, 2002).

Dicha profesionalización debe permitir a la organización educativa un cambio en los paradigmas donde los aspectos formales son prioritarios sobre cualquier otro tipo de relación entre la organización y el individuo, tal como lo refiere Venegas (2001).

El papel del profesional en administración de la educación debe ser el propiciar espacios pertinentes para el beneficio del proceso de enseñanza-aprendizaje, esto a través de modelos de gestión curricular eficientes, debido a que los de corte cuantitativo han producido una ausencia significativa de acciones eficientes en el abordaje de los resultados académicos (Garbanzo y Orozco, 2007). 
En este sentido, Brenes (2008) expresa que la efectividad del trabajo administrativo depende del desarrollo de competencias en el líder, entre ellas destacan el

a) poder de la apreciación para identificar el talento,

b) mantener el equipo enfocado en las tareas más importantes, generar y prolongar la confianza en las acciones organizativas a través de la honestidad, transparencia, buen trato y juego limpio,

c) convertir al equipo en aliados y ser él mismo, un aliado del equipo, esto claro depende de las anteriores competencias.

\section{Gestión de la educación.}

Toda acción que conlleve la puesta en práctica de las funciones determinadas en una organización, se considera dentro del fenómeno de la gestión. La palabra gestión suele traducirse como dirección, organización y/o gerencia (Pozner, 2000), por ello, se hace referencia al término como la acción de facilitar, animar, influir, motivar, entusiasmar y orientar el desempeño y las conductas organizacionales de las personas, en la búsqueda de los objetivos planteados (Sepúlveda, Lagomarsino, Bahamondes y Romero, 2006).

En ese sentido, la gestión implica la actuación de la colectividad y no solo los esfuerzos individuales, por lo cual potenciar las habilidades de las personas resulta fundamental para el logro de los propósitos organizacionales.

Hoy día, las organizaciones se estructuran en función de la actuación principal de quienes laboran en ellas, en la búsqueda de satisfacer sus necesidades. Chiavenato (2009), expresa que es importante que las organizaciones inviertan en las personas que conocen los productos y servicios que ofrecen, con el fin de tener claridad en cómo crearlos, desarrollarlos, producirlos y mejorarlos. Así, el proceso de gestión toma en cuenta las particularidades de cada persona y las habilidades que posee, con el fin de emplearlas en beneficio de la colectividad organizativa.

Hacer realidad los objetivos organizacionales con todo lo que ello conlleva, es la referencia más directa de lo que es la gestión. La administración de la educación requiere de procesos de gestión efectivos que sustenten el ser y quehacer de la labor educativa.

Es por eso, que en el contexto educativo, la gestión se entiende como el ejercicio administrativo que permite el planteamiento de estrategias para la toma de decisiones que mejoren los procesos educativos y la calidad de la educación (Duchi y Andrade, 2001). 
De lo anterior, se desprende que la gestión tiene la obligación de realizar acciones que produzcan beneficios a la comunidad educativa. Al respecto, Izurieta (2010) expresa que "esta concepción privilegia lo pedagógico, lo académico y la gestión del conocimiento sobre lo administrativo y facilita la conformación del cuerpo docente como academia real, crisol del pensamiento" (p. 37).

Con ello, se rescata el interés de gestionar a favor del fin último del campo educativo, el compromiso que adquiere con la sociedad y la organización educativa al proveer las condiciones necesarias para el buen funcionamiento del sistema educativo. Es decir, la gestión se enfoca en la realización correcta de los procesos de enseñanza y aprendizaje.

Así, la gestión de la educación se entiende como el proceso donde, el directivo o equipo directivo, determinan las acciones a seguir, según los objetivos, necesidades, cambios deseados, nuevas acciones solicitadas y la forma como se realizarán estas (Sepúlveda et al., 2006).

Por tanto, la gestión de la administración de la educación vela por el buen desempeño del personal docente y administrativo, en función de los requerimientos de la comunidad educativa en general, y de la consecución de los objetivos organizacionales que trazan el rumbo de acción del centro educativo.

La gestión administrativa, entonces, son todas aquellas acciones que se realizan para la obtención de los resultados propuestos por la organización, por medio de la ejecución colectiva de las personas que la integran, quienes evidencian habilidades y capacidades coherentes con el funcionamiento de la institución educativa, con el fin de mejorar las condiciones para el desarrollo del proceso enseñanza y aprendizaje. Por eso, Vargas (2008) indica que la gestión educativa, vista a través de la teoría organizacional, se entiende como un conjunto de procesos teórico-prácticos integrados, horizontal y verticalmente dentro del sistema educativo, para cumplir los mandatos formales, bajo nuevas formas de comprensión y conducción de las organizaciones escolares.

\section{Gestión del talento humano.}

Como parte fundamental de las organizaciones educativas, la gestión debe focalizar su atención en el recurso humano que la hace realidad. De ahí, que la gestión del talento humano sea la función que permite la colaboración eficaz de las personas para el alcance de los objetivos organizacionales e individuales (Chiavenato, 2009).

Desde esta perspectiva, se torna indispensable que sean las características individuales al servicio de la colectividad, las que intervengan por el bien más preciado del sistema educativo, 
la población estudiantil. La organización debe reconocer que su trabajo va más allá de la realización de una actividad; más bien, implica mística y visión en sus actos para impactar a la población meta a la que se dirige.

Para que exista una buena colaboración por parte de la comunidad educativa, es indispensable que los objetivos de la gestión se orienten por las siguientes características, que Arroyo (2007) expresa como:

1) Determinar deberes, derechos y la naturaleza de los puestos en la organización.

2) Prever la necesidad del personal para períodos y tiempos lectivos.

3) Atraer a interesados en ocupar puestos en la organización.

4) Seleccionar a los candidatos para el logro de los objetivos organizacionales.

5) Incorporar al personal en la doctrina de la organización.

6) Proporcionar al personal, la experiencia básica que requiere para su desempeño efectivo.

La relevancia de considerar lo expuesto, permite pensar en una gestión del talento humano, acorde con las necesidades y requerimientos de la educación, en la actualidad, lo que permite entrever opciones educativas enriquecidas por el personal docente y administrativo que lleva a cabo las acciones de gestión.

\section{Deserción}

La ausencia de la población estudiantil en las aulas por diversidad de causas, es un elemento constante en la realidad de los centros educativos, lo que provoca la frustración de la formación académica requerida para el desenvolvimiento pleno de las personas en sociedad. Elías y Molina (2009) expresan que la deserción escolar es un proceso de alejamiento paulatino de la escuela, que culmina con el abandono por parte del niño, niña o adolescente. El fenómeno visualizado como proceso, plantea la necesidad educativa de determinar el nivel de alejamiento en que el adolecente se encuentra, con el fin de buscar la mejor forma de insertarlo, nuevamente, en el sistema educativo.

En condiciones normales, la deserción se manifiesta en diferentes etapas que indican, poco a poco, la realidad que se gesta en la población estudiantil. Por eso, antes de que 
se presente la deserción física, suele suceder que los jóvenes han desertado del conocimiento y aunque muchos se quedan en la institución, lo hacen por ser el lugar de encuentro con sus pares (Cardona, 2002, citado por Castro y Rivas, 2006). De ahí, que el fenómeno responda a ciertos indicadores que la organización educativa, en su gestión administrativa, es importante que considere estudiar para tratar de disminuir sus efectos.

En esta dirección, Elías y Molinas (2009) mencionan que la deserción conlleva una serie de pasos; es decir, se da un inicio, un desarrollo y durante este, se desencadena la última decisión, el abandono del sistema educativo. Es fundamental que este proceso histórico de la deserción, se catalogue en los diferentes casos que se presentan en las instituciones educativas, pues la diversidad de factores que inciden en él marca la diferencia en la manifestación de este.

Al relacionar el fenómeno como una decisión, Álvarez, Arias, Cortez, Montiel, Rosales y Umaña (2006) coinciden en que la deserción se relaciona con manifestaciones de la conducta que podrían ser el resultado de la interacción de diferentes variables, las cuales desembocan en el abandono de las actividades académicas. Esa decisión, tal y como lo expresa Rojas (2000), se determinada por cambios propios de la adolescencia, entre ellos los aspectos físicos, emocionales y otros que provocan inestabilidad y rechazo hacia sus estudios.

Lo anterior, hace a la administración de la educación iniciar un proceso de control de los múltiples causas que propician la deserción (Elías y Molinas, 2009); en esta dirección, Ruiz (2006) expresa que dentro de los factores de deserción se encuentra socioeconómicos, contextuales y los relacionados al currículo. Razón por la cual el conocimiento de los elementos esenciales que dan lugar al abandono de la academia, permite una reacción más pertinente con las necesidades educativas de la juventud en la adolescencia.

\section{Factores asociados a la deserción escolar}

Castillo, Clapes, Corominas, Ramón y Tubilleja (2006), definen los factores que propician la deserción con respecto a la relación, influencia y cómo acumulan y complican cada caso en particular. La población estudiantil-como se ha mencionado-convive con eventos cuyas relaciones acumulan sensaciones negativas de sus posibilidades de culminar con éxito su experiencia escolar complicando su permanencia en el sistema educativo.

Para la sociedad costarricense algunos de estos factores, por los que la juventud toma la decisión de desertar, se debe a la necesidad de trabajar, la falta de apoyo familiar, falta de motivación y aburrimiento, la poca pertinencia de los métodos de enseñanza y aprendizajes, falta de capacitación y sensibilización de los docentes y un débil apoyo del gobierno (Instituto de Estudios Sociales en Población, 2006). 
Por su parte, el Programa Estado de la Nación en Desarrollo Humano Sostenible (2008) identifica como los problemas del sistema educativo que inciden en la "desescolarización", las discontinuidades en los ciclos educativos, los problemas de rendimiento académico, la debilidad de los programas de acción afirmativa, la escasa diversificación, la baja calidad y pertinencia de la educación, entre otros.

Así, el MEP alude a que también debe considerarse la repitencia como uno de los principales causantes de la deserción escolar, en el nivel nacional. En este sentido, no se puede descartar que los parámetros bajo los que se da la repitencia sean resultado de la influencia de misma institución educativa.

En este sentido Purzzo (2003), expresa que acciones que se aplican a nivel institucional produce fracaso estudiantil a través de situaciones didácticas-pedagógicas y las secuelas que trae consigo en el estudiante, las cuales refieren sufrimiento y frustración.

Al respecto, Gonzales (2006) expresa ciertas prácticas empujan a la población estudiantil a abandonar el sistema educativo. Entre ellas están: horarios inflexibles, currículos fragmentados, estrategias de enseñanza y materiales inadecuados, evaluaciones competitivas, creencias y actitudes de los profesores hacia el alumnado y sus familias, entre otras razones.

Por tal razón, es importante que la organización preste un especial interés en velar porque las situaciones descritas no se manifiesten y, por ende, que la organización educativa propicie condiciones para la movilidad social y no de exclusión (Bourdieu y Passeron, 1970), que realice su gestión sin ampliar las brechas sociales existentes en la sociedad.

\section{Consecuencias de la deserción escolar}

Para el sistema educativo, el fenómeno de la deserción implica un desperdicio de recursos; para la sociedad en general, impacta la competitividad económica y la cohesión social. En esta misma idea, Ruiz (2006) expresa que la deserción en sí, constituye un problema para el sistema educativo costarricense ya que debilita las posibilidades de desarrollo del país.

En el aspecto económico una de las razones primordiales por la que la población estudiantil deserta del sistema educativo: es la búsqueda de trabajo. Sin embargo, las opciones de empleo no llegan con facilidad, porque aún no poseen los conocimientos para ser mano de obra productiva, por lo que aparece un alto riesgo de vagancia y vandalismo para la juventud que abandona sus estudios (Rojas, 2002).

Al respecto, Roman (2009) presenta estimaciones del Banco Interamericano de Desarrollo (BID), donde en Latinoamérica un trabajador con seis años de escolaridad en su primer empleo 
logra un salario por hora $50 \%$ más que quien no tiene y si esta es de 12 años la distancia aumenta a $120 \%$ y si esta llega a 17 años lo supera en un $200 \%$.

\section{Estrategias para la prevención de la deserción escolar y la retención estudiantil.}

Aunado a las estrategias que disminuyen la deserción escolar, se encuentran las acciones catalogadas como de prevención del fenómeno y la retención de la población estudiantil en las aulas escolares.

Diez de Castro, García del Junco, Martín y Periañez (2001) se refieren a la prevención como la capacidad de anticipar los problemas que pudieran presentarse en el futuro, al tiempo que se diseñan los mecanismos oportunos para hacerles frente. Es decir, son todas aquellas acciones que se establecen para prever que sucedan casos de deserción en las distintas instituciones educativas.

Por su parte, la OEA (2003) expresa que la retención escolar es entendida como la "capacidad del sistema educativo para lograr la permanencia de los alumnos en las aulas, garantizando la terminación de ciclos y niveles en los tiempos previstos y asegurando el dominio de las competencias y conocimientos correspondientes" (p. 19).

Para lograr la retención estudiantil en los centros educativos, por tanto, debe existir una adecuada planificación, ya que sin ésta aumenta la en los sistemas educativos, especialmente cuando la población estudiantil ingresa sin una orientación vocacional. Siendo que, determinar el problema es un avance en la solución del mismo, la retención estudiantil en las aulas es un desafío a superar para las organizaciones educativas, por lo que es de vital importancia la detección de casos y los factores que lo despliegan, con el fin de atenderlo de la manera más adecuada.

En este sentido, se proponen una serie de estrategias para prevenir la deserción e incrementar la capacidad de retención del alumnado en las instituciones educativas. A saber, estas estrategias son:

1) Demostrar altas expectativas por el desempeño estudiantil, de modo que sientan la motivación por los resultados de sus esfuerzos.

2) Programas de apoyo académico, social y personal.

3) Retroalimentación temprana a la población estudiantil para la identificación de sus debilidades, con el fin de disponer del tiempo necesario para corregirlas y superarlas. 
4) Valorar la integración de la población estudiantil a la comunidad educativa, a través del contacto directo y de calidad con el personal docente, administrativo $y$, en general, con sus propios pares.

5) Ambientes que incentiven el aprendizaje, por medio de la incorporación de nuevas tecnologías de información y comunicación, prácticas de docencia innovadoras, cambios en métodos de enseñanza y aprendizaje, entre otros. (Universidad de Chile, 2008, p. 53)

Todas las estrategias mencionadas deben ser atendidas por la organización educativa, quien realiza la adaptación de las mismas a la realidad de su comunidad, de acuerdo con sus requerimientos y necesidades. Es importante prestar atención a las percepciones de las personas involucradas en este proceso, para dar paso a estrategias más pertinentes y claras en el objetivo que se pretende alcanzar: mejorar el rendimiento de las acciones implementadas para la prevención de la deserción y retención de la población estudiantil del centro educativo.

\section{Marco metodológico}

Los objetivos de esta investigación plantearon la necesidad de establecer una relación cercana con los sujetos de estudio, en el propio lugar donde se presentan los hechos a estudiar. Por esta razón, de acuerdo con el planteamiento del problema de la investigación, se considera de tipo descriptivo, pues se especifican las propiedades importantes de personas, grupos, comunidades tales como visiones, conocimientos y percepciones del fenómeno (Barrantes, 2006).

Al respecto, Hernández, Fernández y Batista (2006), expresan que este tipo de investigación es útil para mostrar con precisión las dimensiones de un fenómeno, por lo cual el investigador debe definir o al menos visualizar qué se medirá, siendo una de sus ventajas que permite hacer predicciones aunque sean incipientes.

Es importante, para efectos de esta investigación que aún cuando se cuenta con informes detallados elaborados por el departamento de orientación donde la población estudiantil expresaba los motivos de su deserción; los objetivos de ésta se centraron en el análisis de las estrategias para la prevención de la deserción y retención de la población estudiantil desde la perspectiva del personal docente y administrativo.

Este análisis se realizó tomando en cuenta tres variables a estudiar, ellas son: 
- Factores que propician la deserción escolar.

- Estrategias para la prevención y retención de la deserción escolar aplicadas en la institución.

- Eficiencia de las estrategias para la prevención de la deserción escolar y retención estudiantil.

La investigación se realizó en el Liceo de Miramar, perteneciente a la Dirección Regional de Educación de Puntarenas, con una población de 65 personas entre personal docente y administrativo, quienes corresponden a la totalidad de la población funcionaria del centro educativo (55 personas docentes y 10 personas funcionarias administrativas).

A los participantes se les aplicó un cuestionario, con el objetivo de identificar el grado de conocimiento de la población en estudio acerca de los factores que propician la deserción escolar, las estrategias para la prevención y retención de la población estudiantil, además de la eficiencia de esas estrategias, de acuerdo con la percepción que poseen los participantes en este trabajo.

El mismo, fue contestado por 61 personas, entre personal docente y administrativo, tal como se presenta en la siguiente tabla.

Tabla 1

Personal docente y administrativo del Liceo de Miramar participantes del estudio

\begin{tabular}{ccccc}
\hline Docentes & Administrativos & Docente enseñanza especial & Analista Sistema & Psicóloga \\
\hline 51 & 5 & 3 & 1 & 1 \\
\hline
\end{tabular}

Fuente: Nomina del Personal docente y Administrativo del Liceo de Miramar.

Además, se utiliza la entrevista semiestructurada para conocer la perspectiva de la la dirección del Liceo donde se realiza la investigación, acerca de los factores que propician la deserción y las acciones que se aplican en la institución educativa.

Otra de las técnicas aplicadas es el grupo focal, para el cual se elabora un reporte en el que se incluyen datos de los participantes, la percepción de los participantes a las interrogantes planteadas para lo cual se contó con una bitácora de la sesión (Hernández et al., 2006). 
El grupo fue compuesto por seis profesionales de distintas especialidades, esta técnica se realizó en una sesión, destacan áreas como: dos docentes del departamento de orientación, dos profesores del departamento de matemáticas, una profesora del departamento español y una profesora de enseñanza especial.

La escogencia para conformar el grupo focal sefundamentó en las siguientes características:

1) Disponibilidad del horario, para participar en la investigación.

2) Apertura para propiciar información necesaria para la investigación.

3) Experiencia reconocida por diversas personas, en relación la aplicación de estrategias para la prevención de la deserción escolar y retención de la población estudiantil en las aulas.

4) Profesoras del departamento de orientación.

5) Profesores con un buen rendimiento porcentual en bachillerato.

6) Profesores con un alto porcentaje de reprobación en los grupos.

\section{Análisis de la información}

Una vez recolectada la información, se procedió a tabular los datos suministrados en el cuestionario por los sujetos de estudio, para determinar el comportamiento de las categorías de la investigación. El análisis de la información se relacionó, además, con las teorías desarrolladas en el marco teórico y los objetivos que guían la investigación.

En el transcurso del desarrollo del análisis, se aprovechó la información proveniente de la entrevista realizada a la dirección y los datos del grupo focal que se llevó a cabo con el personal docente y administrativo seleccionado.

Así, se realiza la triangulación de métodos de recolección de información (cuestionario, entrevista y grupo focal), sustentados en la teoría que respalda el estudio realizado sobre estrategias para la prevención de la deserción escolar y retención de la población estudiantil en el Liceo de Miramar, para la realización del análisis respectivo.

La siguiente figura, muestra la opinión de los participantes en cuanto al bajo rendimiento académico estudiantil como causa de la deserción escolar. 


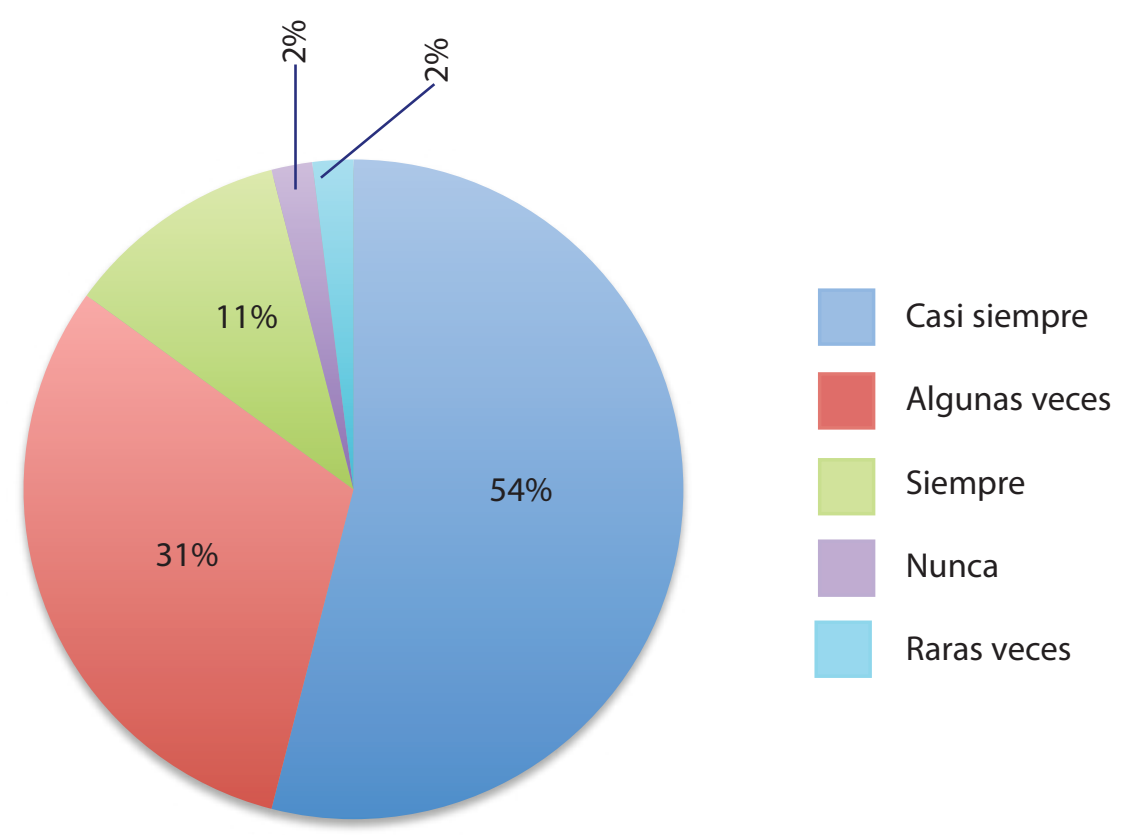

Figura 1. Bajo rendimiento académico estudiantil.

Fuente: Cuestionario aplicado a docentes y administrativos del Liceo Miramar.

Para el Programa Estado de la Nación en Desarrollo Humano Sostenible (2008), el bajo rendimiento de la población estudiantil es uno de los aspectos fundamentales que motivan a la deserción; aspecto que pareciera ser ratificado por los participantes, ya que en el gráfico anterior, el $96 \%$ del personal docente y administrativo considera, en algún grado, que el bajo rendimiento es un indicador de deserción en la población adolescente. Al respecto, la dirección menciona que: "Esto obedece a que los jóvenes, no tienen motivación hacia el estudio".

La anterior afirmación, se observa en el "Informe de deserción del Liceo de Miramar 2010", construido por el departamento de orientación, donde un $80 \%$ de la población desertora expresan que se marchaban por el bajo rendimiento. De ahí, que este indicador debe tomarse en cuenta cuando se piensa en deserción (Moreira, 2007).

Por su parte, el grupo focal expresa que la juventud no tiene porque luchar dado que todo se le da, e incluso las becas la utilizan para comprar lo que necesitan. En ese sentido, mencionan que: "Los jóvenes hoy poseen cualquier cosa que necesitan, no tienen por qué esforzarse e incluso ellos administran sus becas satisfaciendo sus lujos". 
La figura 2, refiere a la aplicación de métodos de enseñanza y aprendizaje inadecuados, por parte del personal docente, como causa de la deserción escolar.

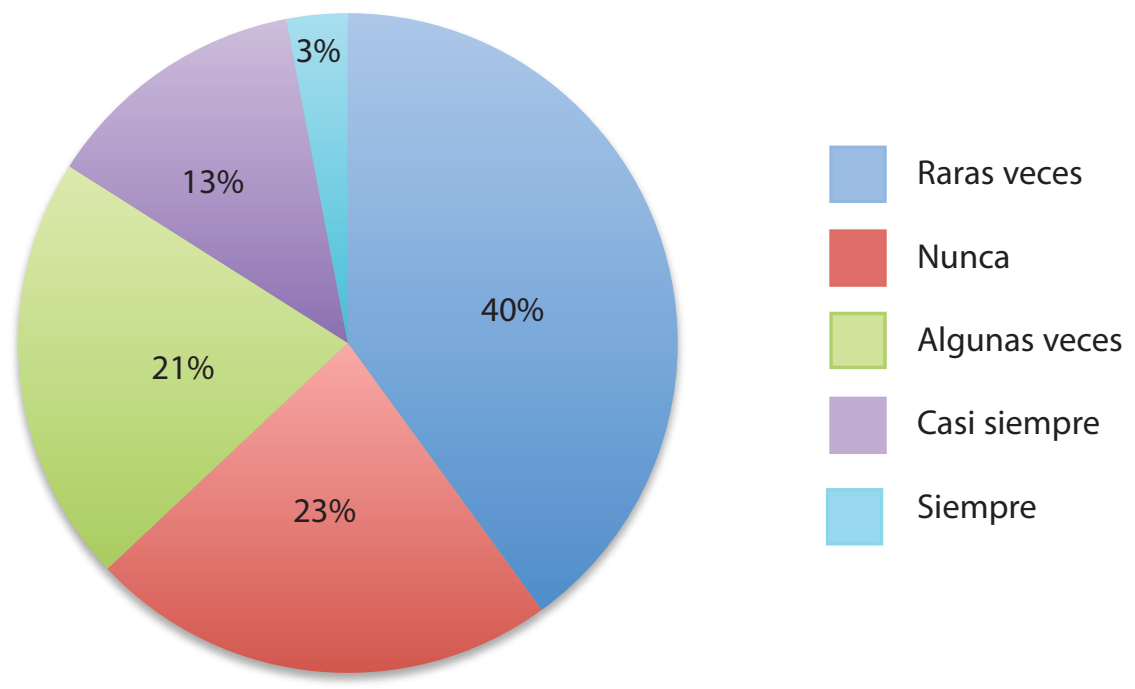

Figura 2. Métodos de enseñanza y aprendizajes inadecuados

Fuente: Cuestionario aplicado a docentes y administrativos del Liceo Miramar.

En razón de lo anterior, Rojas (2000) expresa que para mejorar los índices de deserción se debe regenerar el proceso de enseñanza-aprendizaje, por lo cual adquiere importancia el modificar la manera de organizar, promover y evaluar los aprendizajes básicos, en ese sentido. Al contrario, el personal docente y administrativo opina, en un 53\%, que los métodos de enseñanza que se aplican en la institución no son un factor relevante para que la población estudiantil considere desertar del servicio educativo.

En el grupo focal, no obstante, se externaron comentarios del estudiantado en relación con las clases que reciben, lo que hace pensar que los métodos que se utilizan en el proceso de enseñanza y aprendizaje sí son inadecuados y poco atractivos. Así, el personal administrativo opina que: "Los estudiantes mencionan que las clases son aburridas, sin explicación alguna; no comprenden por qué se imparten".

Así mismo, la población desertora hace referencia a este aspecto como determinante para perder interés en las lecciones que tienen que recibir.

Es obligación de la administración de la educación la búsqueda de asesoramiento y orientación para el personal docente, en aspectos curriculares y administrativos, procurando la incorporación de conocimientos actualizados e innovadores para el beneficio del estudiantado. 
La figura 3, refiere a la causa de deserción que se enfoca en la identificación de posibles estudiantes en riesgo, en las reuniones de personal docente que se llevan a cabo.

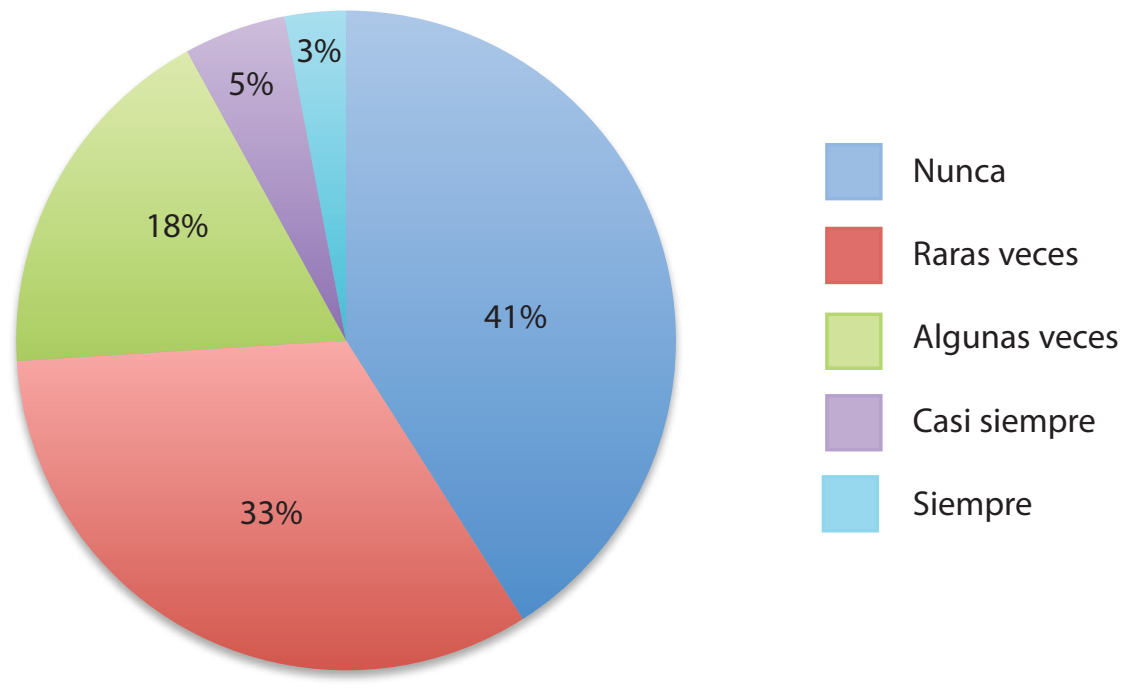

Figura 3. Identificación de posibles estudiantes en riesgo en los consejos de profesores

Fuente: Cuestionario aplicado a docentes y administrativos del Liceo Miramar.

Al respecto, se presentan datos que indican que en la institución no se realizan consejos o reuniones de personal docente para identificar posibles estudiantes en riesgo de deserción, afirmación que es determinada así, por 75\% del personal en estudio.

En el grupo focal, se expresa que estas estrategias no se aplican y que cualquier acción que la institución realiza, siempre es posterior a que el estudiante se ha marchado del centro educativo y del sistema. La dirección comenta, en ese sentido, que existen acciones que, individualmente, ciertos docentes comprometidos con su profesión realizan, por lo que expresa que: "Hay docentes que asignan trabajos extras a estudiantes con bajo rendimiento académico; además, se preocupan por buscar servicios de comedor gratuito y otros, si se sabe que los estudiantes son de escasos recursos. Algunos también, dan centros de su materia".

La administración de la educación debe descentralizar las acciones y extender la responsabilidades pedagógicas para generar pertinencia con el contexto, las redes de la pertinencia operarían a favor de la inserción y permanencia en el sistema educativo (Castro y Rivas, 2006), razón por la cual en el diseño de acciones en contra la problemática es vital la identificación de la población en riesgo. 
En ese sentido, ante la consulta sobre un diseño de perfil de la población estudiantil en situaciones de riesgo de deserción, la figura 4 presenta los siguientes datos.

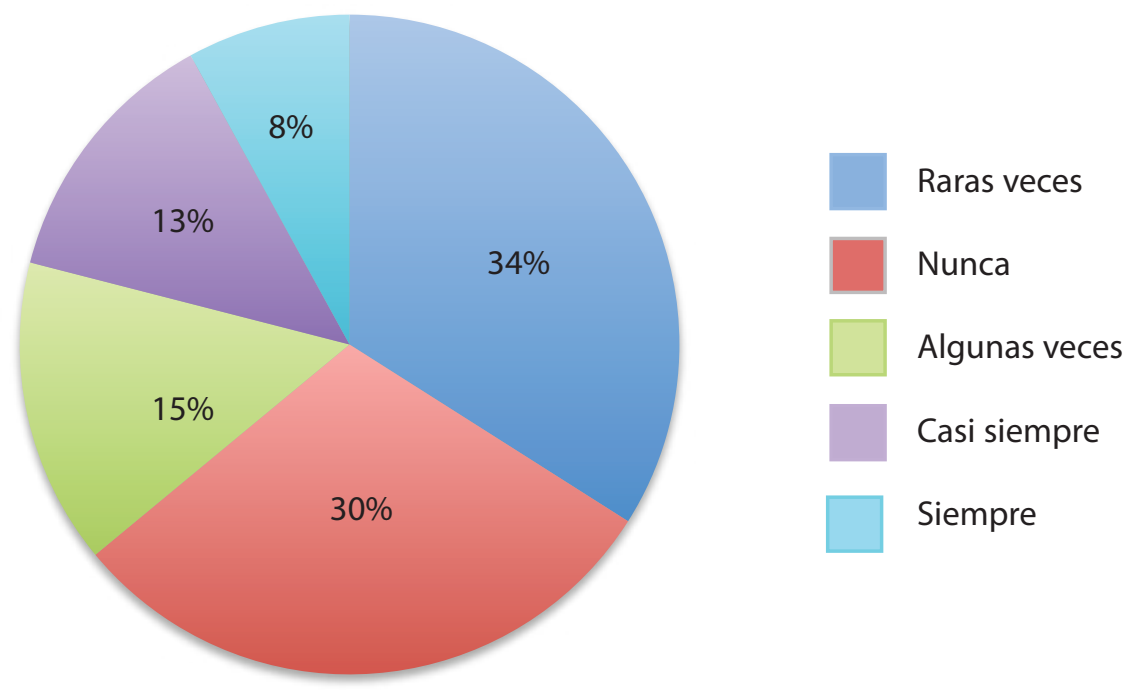

Figura 4. Diseño de un perfil de estudiantes en situaciones de riesgo de deserción

Fuente: Cuestionario aplicado a docentes y administrativos del Liceo Miramar.

En ese sentido, es posible predecir cuando la población estudiantil está en riesgo de abandonar los estudios, por lo que un sistema de alerta temprana para identificar los indicadores de la deserción como la inasistencia frecuente, la repetición, la sobre edad, el bajo rendimiento y los problemas de conducta entre otros, sería un gran apoyo para el seguimiento de este fenómeno educativo, para determinar la ayuda específica para cada estudiante.

Del gráfico, anterior, se concluye que el personal docente y administrativo considera, en su mayoría, que en la institución no se diseñan perfiles estudiantiles en riesgo, lo cual es reiterado por las profesionales en orientación en el grupo focal, quienes expresan que: "No existe ningún diseño que represente, con indicadores, a los estudiantes que está en riesgo de deserción. Solamente se tiene como insumo el informe de deserción institucional".

El "Informe de deserción del Liceo de Miramar 2010", solo muestra algunos de los factores relacionados con la deserción, de manera individual, entre los cuales se encuentra la desmotivación, el bajo rendimiento y el ausentismo.

La dirección, en la entrevista que se le realizó, reconoce que la institución no diseña un perfil de deserción para su detección temprana y retención en el sistema, pero que si elabora el informe mencionado para tener datos concretos de por qué se fue esa población estudiantil en específico. 
Los profesionales en administración de la educación deben transformar los insumos para responder a las demandas de quienes reciben el servicio educativo (Arroyo, 2007), por lo que conocer la población en riesgo de deserción a través de un material diseñado con intencionalidad de la organización, le reconoce su razón de ser.

La siguiente figura, por su parte, muestra la opinión del personal docente y administrativo, en relación con la aplicación de estrategias para la prevención de la deserción y retención estudiantil.

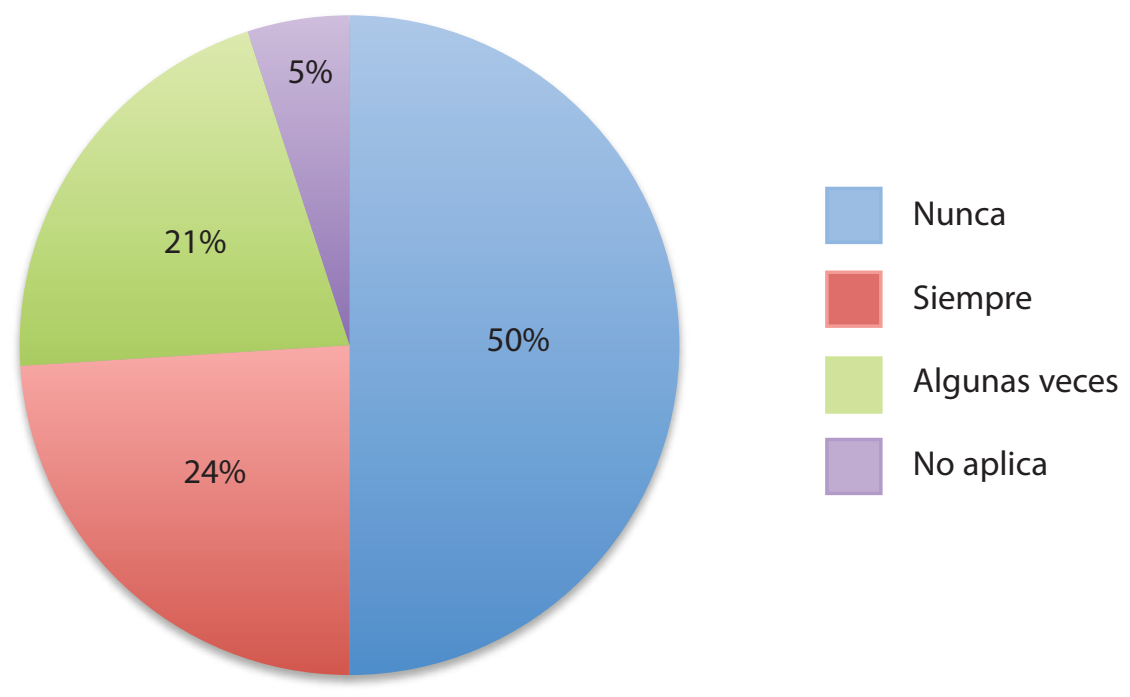

Figura 5. Aplicación de estrategias para la prevención de la deserción y retención estudiantil

Fuente: Cuestionario aplicado a docentes y administrativos del Liceo Miramar.

La percepción del personal docente y administrativo, en ese sentido, indica que en su mayoría, no se aplican o se llevan a cabo solo algunas veces, acciones que se orientan a la prevención de la deserción estudiantil o a la retención en los centros escolares, lo que se representa con un $95 \%$ de los participantes en el estudio.

Esa afirmación, se ratifica por medio de las opiniones manifestadas en el grupo focal, al mencionar que no existe en el Liceo de Miramar un diseño en función de la prevención y retención de la población desertora. Eso se debe a la dificultad que existe para obtener los permios correspondientes para ejecutar acciones estratégicas que motiven e inserten al estudiantado en el quehacer educativo. Al respecto, la profesional en orientación de la educación manifiesta que: "El proyecto que maneja en la actualidad el MEP se llama "El cole en nuestras manos". Para nosotros, existe una contradicción, ya que este proyecto procura que el estudiante quiera estar en la institución. Sin embargo, obtener los permisos para la creación de 
espacios con actividades culturales, académicas, musicales o deportivas, requiere de todo un trámite burocrático en otras instancias de la que lo promueve".

De igual forma, la profesional en administración de la educación indica que, en torno al proyecto del MEP: "En el papel, el proyecto dice una cosa, pero en la aplicación hay que realizar trámites para obtener permisos que se supone, el proyecto mismo debería tener".

La administración de la educación, conociendo que enfrentarse a un fenómeno multidimensional como la deserción implica el uso de estrategias globales y sistémicas, en reformas profundas que tengan en cuenta las demandas sociales del futuro y un esfuerzo sostenido a lo largo de varios años (Marchesi, Pérez, Kovacs, Puig y Hernández, 2003), genera un proyecto que requiere de muchos esfuerzos para que rinda lo que se espera de él, para la retención estudiantil en el sistema educativo.

Así, la siguiente figura refiere la opinión del personal docente y administrativo, en relación con la poca utilidad de las estrategias que se aplican para la prevención de la deserción y retención estudiantil.

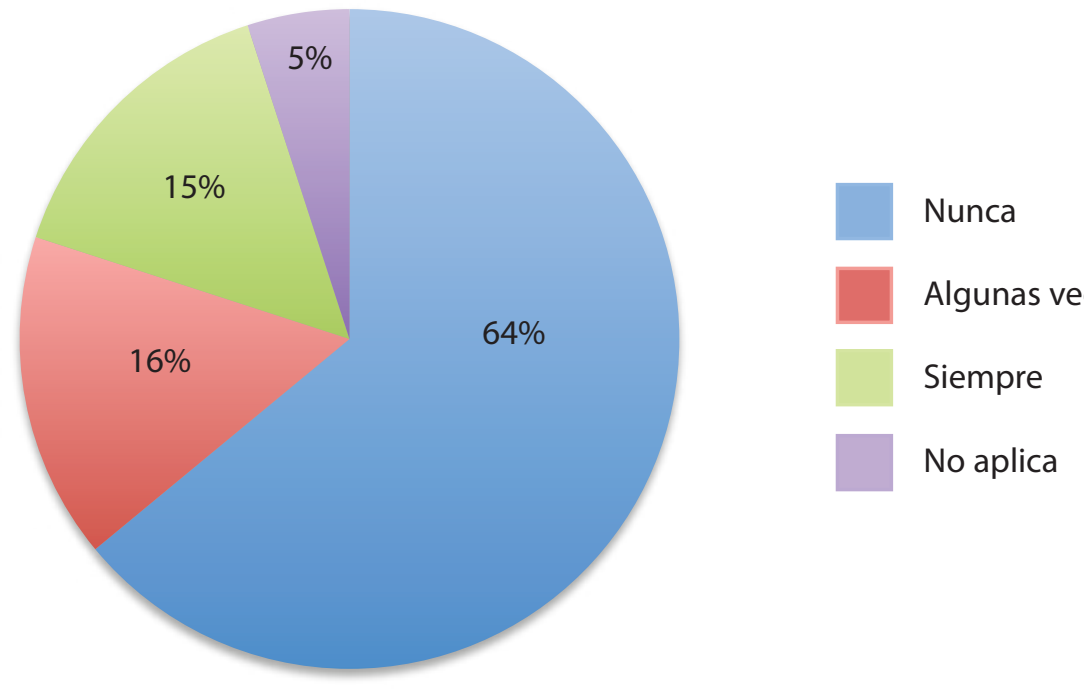

Figura 6. Poca utilidad de las estrategias para la prevención y retención de la deserción.

Fuente: Cuestionario aplicado a docentes y administrativos del Liceo Miramar.

Al respecto, Espindola y León (2002), expresan que la base para la implementación de una estrategia efectiva para la prevención de la deserción, es informar a la comunidad educativa y a la sociedad en general, sobre el problema que se vive en ese sentido. 
Bajo este concepto, pareciera ser que, en el Liceo de Miramar, la comunidad educativa no está informada acerca de la problemática del fenómeno en estudio, ya que un $80 \%$ del personal docente y administrativa expresa que las estrategias que se aplican son de poca utilidad. En el grupo focal, el personal docente manifiesta que esas estrategias no corresponden directamente, al tratamiento de la deserción, al decir que:"No existe una educación a nivel institucional sobre la aplicación de estrategias para la prevención y la retención escolar".

Ante lo anteriormente manifestado, la dirección del centro educativo comenta que: "A nivel institucional contra la deserción, solo lo que el MEP asigna, lo cual es poco".

De esta forma, en el nivel institucional los profesionales en administración de la educación deben asesorar y orientar al personal docente en aspectos curriculares y administrativos, procurando la incorporación de conocimientos que concienticen a la institución de la problemática y luego, la búsqueda de acciones contra ella.

De ahí, que la figura 7 determina las acciones realizadas en materia de prevención, en cuanto al interés del personal docente y administrativo de colaborar con la población estudiantil para que no abandonen el sistema escolar.

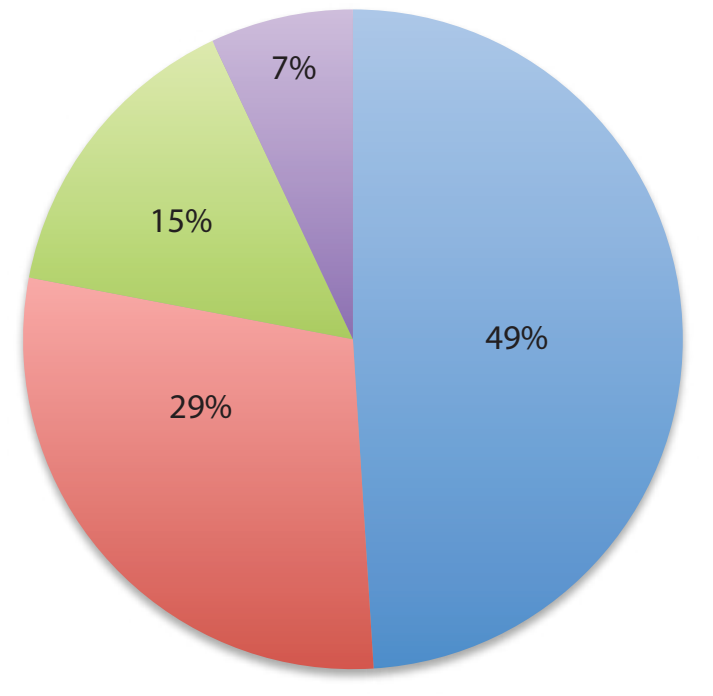

Siempre

Algunas veces

Nunca

No aplica

Figura 7. Acciones realizadas en materia de prevención: más normativas que generadas por el interés de ayudar al estudiante

Fuente: Cuestionario aplicado a docentes y administrativos del Liceo Miramar. 
Aún cuando en la institución existen acciones estratégicas como la asignación de becas y el inicio del proyecto "El cole en nuestras manos", se comprende que estas se llevan a cabo por directrices del MEP y no propuestas por la misma organización educativa. Así, el personal docente y administrativo, considera que las acciones son realizadas por obligación y no por un planeamiento sistemático que esté en función del bienestar de la población estudiantil.

Lo anterior, se reitera con la opinión de los docentes participantes en el grupo focal, quienes expresan que: "La información no se utiliza, sino que se almacena o solo sirve para dar informes. La institución no conoce la realidad del estudiante, ni cuáles son los factores que le hacen desertar; además, complicamos las cosas creando reglas para que el estudiante tenga que cumplir y no nos preocupamos porque cumpla las que existen".

En relación con lo anterior, la dirección manifiesta que: "Las acciones que se aplican son las que solicita el MEP".

La administración de la educación ha evolucionado y su gestión se centra en las actividades prácticas, las cuales a través de la deliberación, toma de decisiones responsable, permite a al personal docente de los centros educativos asumir un papel activo en la construcción de una comunidad (Vargas, 2008).

Aunque tradicionalmente en Costa Rica la administración que se ha utilizado ha sido la normativa, centralizada, no participativa (Venegas, 2001), los desafíos actuales la encaminan a proceso mediante el directivo o equipo directivo, determinan las acciones a seguir e implementan acciones en búsqueda de los cambios demandados (Sepúlveda et al., 2006), por lo cual la gestión debe prestar su atención en el recurso humano, en la gestión del talento que permite la colaboración eficaz de las personas para el alcance de los objetivos organizacionales e individuales (Chiavenato, 2009).

\section{Conclusiones}

A la luz de los datos obtenidos en este proceso de estudio, se concluye para cada uno de los objetivos planteados que:

- De los factores que propician la deserción escolar en el Liceo de Miramar se identifican los siguientes: bajo rendimiento académico y desmotivación de la población estudiantil, poco apoyo por parte de las familias, métodos inadecuados aplicados por el personal docente y el ausentismo estudiantil en las organizaciones educativas.

- En relación con las estrategias para la prevención y retención de la deserción que lleva a cabo la administración de la educación se concluye que: no existe coordinación 
entre las estrategias aplicadas; no existe sistematización de las acciones y éstas, a su vez, carecen de intencionalidad, es decir, no existe planificación que tome en cuenta las características del fenómeno y la mejor forma de enfrentarlo. Además, no existe una concientización, en el nivel institucional de la problemática, sus causas y consecuencias.

- Con respecto a la eficacia de las estrategias para la prevención de la deserción y retención estudiantil que se aplican en el centro educativo se concluye que éstas no son efectivas, ya que son acciones aisladas que desconocen la realidad de la población estudiantil.

Dada las consideraciones anteriores, se diseñan estrategias que tomando en cuenta el contexto de la institución, contribuyan al fortalecimiento de las acciones que se aplican en función del bienestar de la comunidad educativa, en general.

\section{Propuesta de intervención para la atención de la deserción y retención estudiantil en el Liceo de Miramar, Puntarenas}

La administración de la educación, si quiere preparar talento humano para la nueva época que abarca cada vez, más todos los espacios de la vida personal y colectiva, debe en su gestión, hacer uso de todos sus enfoques conceptuales y sus paradigmas actitudinales (Izurieta, 2010).

La administración en su proceso de trabajo, con personas y recursos en búsqueda de alcanzar las metas de la organización, no puede perder de vista, el sentido humano, su razón de ser, es decir trabaja con personas utilizando a las mismas como recurso, por lo cual su realización como ciencia consiste en mejorar los estados actuales educativos de todos sus actores.

La acción educativa está vinculada a la capacidad de impulsar procesos de cambio cultural (Pozner, 2000), para enfrentar el problema de la deserción y en el ámbito operatorio educativo, donde el personal docente, en el corto y en el largo plazo, promuevan prácticas en la población estudiantil que disminuyan la problemática mencionada; así las prácticas educativas pueden provocar un cambio de la cultura burocrática, limitadas a esquemas de programación y reglamentación (Vargas, 2008).

Por ello, la presente propuesta pretende a través de la gestión de la educación, contribuir en la disminución de la deserción estudiantil, mediante acciones estratégicas que creen conciencia en los actores educativos de dicha problemática, proporcionando herramientas eficaces que complementen las ya existentes contra la lucha de este flagelo.

La propuesta se detalla a continuación, en la siguiente figura. 


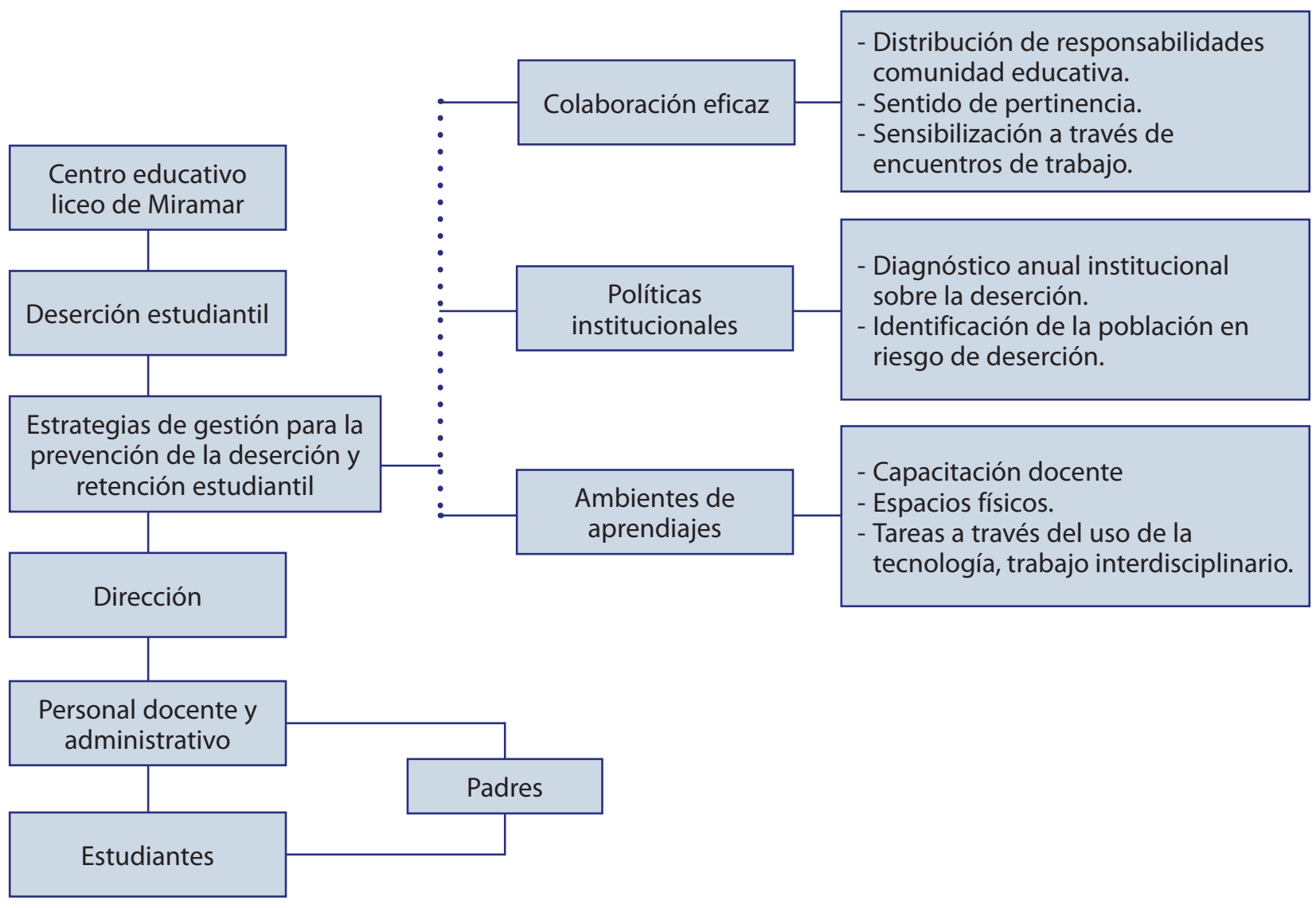

Figura 8. Esquema estructural de la propuesta de intervención para la prevención de la deserción y retención estudiantil en el Liceo de Miramar, Puntarenas

Fuente: Ovares, 2011. Elaboración propia.

La propuesta busca proveer herramientas eficaces en la lucha de la deserción estudiantil, conociendo de la responsabilidad de la administración de la educación para con la sociedad. En este sentido, los esfuerzos se dirigen a tres aspectos teóricos expuestos:

1) la colaboración eficaz de las personas para el alcance de los objetivos organizacionales e individuales (Chiavenato, 2009);

2) el establecimiento de políticas institucionales en la lucha contra la deserción;

3) la creación de ambientes de aprendizaje que favorezca la relación alumno-docenteinstitución. 
El diseño de las estrategias se fundamenta en aspectos esenciales para la prevención de la deserción estudiantil como la definición de responsabilidades, la estructura de acciones para remediar el acto desertor en los centros educativos, la participación activa del personal docente y administrativo; así como el diagnóstico de necesidades estudiantiles.

La elaboración de la propuesta siempre constituyó un fortalecimiento de las estrategias que, de una u otra forma, la institución ya viene aplicando. La misma responde a fundamentos teóricos expresados por la literatura especializada, por lo que busca adaptarse a una realidad específica y aterrizada en acciones que estén dentro de lo que una institución con voluntad quiera realizar.

\section{Referencias}

Álvarez, W., Arias, A., Cortés, C., Montiel, E., Rosales, X. y Umaña, E. M. (2006). Factores asociados a la deserción de la población estudiantil de sétimo año del Liceo José Martí, del Liceo de Chacarita y del Liceo Antonio Obando Chan, en año 2004. (Tesis de Licenciatura). Universidad de Costa Rica. San José, Costa Rica.

Arroyo, J. A. (2007). Gestión estratégica de personal en las organizaciones educativas. San José, Costa Rica: EUCR.

Barrantes, R. (2006). Investigación: Un camino al conocimiento. Un enfoque cualitativo y cuantitativo. San José, Costa Rica: EUNED.

Brenes, L. (2008). Gestión estratégica de la microempresa turística. San José, Costa Rica: EUNED.

Bourdieu, P. y Passeron, J.-C. (1970). La reproduction. París: Editorial de Minuit.

Castillo, M. (Coord.), Clapés, G., Corominas, J., Ramón, E. M.y Tubilleja, E. (2006). Como evitar el fracaso escolar en secundaria. Recursos. España: Narcea.

Castro, B. y Rivas, G. (segundo semestre, 2006). Estudio sobre el fenómeno de la deserción y retención escolar en localidades de alto riesgo. Sociedad Hoy, 11, 35-72. Recuperado de http://redalyc.uaemex.mx/pdf/902/90201103.pdf

Certo, S. (2001). Administración moderna (8ª ed.). Bogotá: Mcgraw-Hill.

Chiavenato, I. (2009). Gestión del talento humano. México: McGraw-Hill.

Programa Estado de la Nación en Desarrollo Humano Sostenible. (2008). Estado de la Educación 2 ( $2^{\mathrm{a}}$ ed.). San José, Costa Rica: CONARE.

Diez de Castro, E., García del Junco, J., Martínez, F. y Periañez, R. (2001). Administración y dirección. Madrid: McGraw-Hill. 
Duchi, M. y Andrade, B. (2001). Los procesos de gestión administrativa y pedagógica del núcleo "Nataniel Aguirre" de Colomi, Cochabamba-Bolivia: Hacia una perspectiva de redes de EIB en Suscal Cañar-Ecuador. (Tesis de Maestría). Universidad Mayor de San Simón, Bolivia. Recuperado de http://bvirtual.proeibandes.org/bvirtual/docs/tesis/proeib/Tesis_ Andrade_y_Melchor.pdf

Elías, R. y Molinas, J. (2009). La deserción escolar de adolescentes en Paraguay. Reformas pendientes en la educación secundaria. Santiago, Chile: Fondo de Investigaciones Educativas.

Espíndola, E. y León, A. (setiembre-diciembre, 2002). La deserción escolar en América Latina: un tema prioritario para la agenda regional. Revista Iberoamericana de educación, 30. Recuperado de http://dialnet.unirioja.es/servlet/articulo?codigo=307359

Garbanzo, G. y Orozco, V. (2007). Desafíos del sistema educativo costarricense:un nuevo paradigma de la administración de la educación. Revista Educación, 31(2), 95-110.

García, N., Rojas, M. y Campos, N. (2002). La administración escolar para el cambio y el mejoramiento de las instituciones educativas. San José, Costa Rica. EUCR.

González, M. T. (2006). Absentismo y abandono escolar: Una situación singular de la exclusión educativa. REICE: Revista Electrónica Iberoamericana sobre Calidad, Eficacia y Cambio en Educación, 4(1), 1-15. Recuperado de http://www.rinace.net/arts/vol4num1/art1.pdf

Hernández, R., Fernández, C. y Batista, P. (2006). Metodología de la investigación (4ª ed). México: McGraw-Hill.

Instituto de Estudios Sociales en Población (IDESPO). (2006). Percepciones de la población costarricense sobre el sistema educativo. Pulso Nacional, 44, 1-18. Heredia, Costa Rica: IDESPO-UNA.

Izurrieta, L. (2010). ¿Administración educativa o gestión educativa? Hacia un nuevo modelo de gobierno del sistema de educación superior. Quito, Ecuador: Consejo Nacional de Evaluación y Acreditación de la Educación Superior del Ecuador.

Kootz, H., Weihreich, H. y Cannice, M. (2008). Administración: Una perspectiva global y empresarial ( $8^{\mathrm{a}}$ ed.). México: Mcgraw-Hill.

Marchesi, A.; Pérez, E. M., Kovacs, K., Puig, J. M. y Hernández, C. (2003). El fracaso escolar: Una perspectiva internacional. Madrid: Alianza Editorial.

Moreira M., T. (2007). Perfil sociodemográfico y académico de estudiantes en deserción del sistema educativo. Actualidades en Psicología, 21(108), 145-165. Recuperado de http:// redalyc.uaemex.mx/src/inicio/ArtPdfRed.jsp?iCve=133212641007 
Organización de los Estados Americanos [OEA]. (2003). Estrategias y materiales pedagógicos para la retención escolar. Recuperado de http://www.slideshare.net/damarysesther/ estrategias-y-materiales-pedaggicos

Pozner, P. (2000). Gestión educativa estratégica. Buenos Aires, Argentina: IIPE/UNESCO.

Purzzo de Di Pego, V. (2003). Biografía del fracaso escolar. Recuperación psicopedagógica. Buenos Aires: Espacio Editorial e Instituto de Ciencias de la Educación para la Investigación Interdisciplinaria

Rojas, M. E. (junio-setiembre, 2000). La deserción escolar en Costa Rica: Un estudio de causas y consecuencias en una institución educativa. Diálogos: Revista electrónica de historia, 1(4), 1-27. Recuperado de http://redalyc.uaemex.mx/pdf/439/43910406.pdf

Román, M. (2009). El fracaso escolar de los jóvenes en la enseñanza media. ¿Quiénes y porqué abandonan definitivamente el liceo en Chile? REICE, 7(4). Recuperado de http://www. rinace.net/reice/numeros/arts/vol7num4/art5_htm.htm

Ruiz, Á. (2006). Universalización de la educación secundaria y reforma educativa. San José, Costa Rica: EUCR.

Sepúlveda, C., Lagomarsino, M., Bahamondes, A. y Romero, J. (2006). Estilos de gestión y administración en educación municipalizada asociados con estrategias del CPEIP para formación continua del profesorado. Santiago, Chile: Universidad Católica Silva Henríquez. Recuperado de http://www.rmm.cl/usuarios/pponce/doc/200704241330070.ucsh_ informe_final_nov_2006.pdf

Vargas, I. (enero-abril, 2008). Análisis de cinco desafíos en el ejercicio de la administración educativa. Revista Actualidades Investigativas en Educación, 8(1), 1-15. Recuperado de http://revista.inie.ucr.ac.cr

Venegas, P. (2001). Retos y perspectivas de la administración educativa en un nuevo milenio. Recuperado de http://estatico.uned.ac.cr/posgradoysociedad/revista/documentos/ REVISTA\%20VIRTUAL/2003/La\%20administraci\%F3n\%20Educativa\%20en\%20el\%20 siglo\%20XXI.pdf

Universidad de Chile. (2008). Informe final "Estudio sobre causas de la deserción universitaria". Santiago, Chile: Centro Microdatos, Departamento de Economía. Recuperado de http:// www.oei.es/pdf2/causas-desercion-universitaria-chile.pdf 